\title{
Cementless Femoral Neck Endoprosthesis Spiron in Men in Aspects of Clinical Status and Quality of Life in Average a 5-year Follow-up
}

\section{Tomasz Stołtny}

District Hospital of Trauma Surgery

Jarosław Pasek ( $\nabla$ jarus_tomus@o2.pl)

Faculty of Health Sciences Jan Długosz University in Częstochowa https://orcid.org/0000-0001-6181$337 X$

\section{Michał Pyda}

District Hospital of Trauma Surgery

\section{Daniel Spyrka}

District Hospital of Trauma Surgery

\section{Michał Białek}

District Hospital of Trauma Surgery

\section{Bogdan Dugietto}

University of Silesia in Katowice

\section{Wojciech Gąsior}

X-Rehab Centre of Rehabilitation

\section{Michał Czarnecki}

X-Rehab Centre of Rehabilitation

\section{Bogdan Koczy}

District Hospital of Trauma Surgery

\section{Research article}

Keywords: femoral neck endoprosthesis, hip osteoarthritis, avascularnecrosis, HHS, WOMAC-HIP, SF-12, Quality of Life

Posted Date: July 1st, 2020

DOI: https://doi.org/10.21203/rs.3.rs-39182/v1

License: (c) (1) This work is licensed under a Creative Commons Attribution 4.0 International License.

Read Full License 


\section{Abstract}

Background: Hip arthroplasty is increasingly performed in young people actively playing sports, professionally active, or treated with steroid drugs in the course of autoimmune diseases. Significantly more often in this group of patients the cause of osteoarthritis is sterile femoral head necrosis. Femoral neck endoprosthesis can be an alternative to pituitary or classical cementlessarthroplastyin the surgical treatment of osteoarthritis.

Material and methods: The study group consisted of 27 men aged 32 to 65 years (average 46 years) in whom femoral neck endoprosthesis was implanted (16 on the left side, 11 on the right side).The most common indications for surgical treatment were idiopathic osteoarthritis of the hip (19 patients) and arthrosis due to sterile femoral osteonecrosis (8 patients). Operational access according to Hardinge in the patient's position on the back by embedding a uncemented femoral neck endoprosthesis in ceramic articulation was applied.In the studied group of patients, clinical condition was assessed using the HHS, WOMAC-HIP classification. An assessment of the quality of life was also carried out on the SF-12 scale and pain assessment on the VAS scale.Analyzes were performed before surgery, while the control test was carried out on average over a 5-year observation period.

Results: The clinical condition of the examined men on the HHS (49.81 vs 84.05) and WOMAC-HIP (40.59 vs 13.5) scale significantly improved $(p<0.05)$ in relation to the value before surgery.Similar results were obtained in the assessment of quality of life on the SF-12 scale (9.89 points vs. 32 points); $(p<0.05)$ and pain on the VAS scale $(6.74$ vs 0.58$) ;(p<0.05)$ during follow-up.

Conclusions: The use of femoral neck endoprosthesis in men with hip arthrosis in the mean follow-up of 5 years improved both the clinical condition and the quality of life of the operated patients while reducing the pain.

\section{Introduction}

Osteoarthritis is currently the fourth leading cause of disability in women and the eighth in men. Among the most common causes of osteoarthritis of the hip due to sterile femoral head necrosis are: chronic dialysis, femoral neck fracture, hip inflammation, immunosuppression in organ transplantation [1].Sterile osteonecrosis of the femoral head, as the second cause of osteoarthritis in young patients, is almost 10 times more common in the male population than earlier research results might suggest.This disease at such a young age is the reason for giving up physical activity, absenteeism from work and often is found in patients undergoing treatment for a "different" disease [1,2].

Despite significant development of pharmacotherapy, inoperable treatment, especially in advanced forms of the disease, does not bring satisfactory results. In such cases, the only solution is arthroplasty. The hip arthroplasty is intended to pursue the philosophy of 'prosthesis before prosthesis'.In a situation of destabilization, it should successfully allow implantation of a pituitary or classic cementlessendoprosthesis. In such a young group of patients, surgical methods that "save bone tissue" 
are constantly sought, which, having regard to the clinical condition, pain, will significantly reduce them, which will allow operated patients to return to physical, professional and sports activities[3,4].

For the first time, total hip arthroplasty with a polyethylene cup was made in 1962 at Sir John Charnley Clinic in Wrightington. The philosophy of replacing the affected joint with available implants has not changed since then.However, the enormous development of materials and surgical techniques significantly extends the time of stable implantation of endoprosthesis components and reduces the risk of revision surgery.This allows the orthopedic physician in many selected cases to decide on the implantation of a femoral neck endoprosthesis in ceramic articulationin a patient under forty. $[2,5]$.

\section{Aim of the study}

The aim of the study was pre- and postoperative clinical evaluation, quality of life analysis and painassesmentin men with advanced osteoarthrosis after femoral neck endoprosthesis in full ceramic articulation.

\section{Material And Methods}

In the years 2012-2016, in the Department of Trauma and Orthopedics of the Provincial Hospital of Traumatic Surgery in xxxxxx, 50 femoral neck endoprosthesis in men were performed.27 patients volunteered for a follow-up examination; including 16 operated on the left side and 11 on the right side.The average age of patients was 46 years (from 32-65 years). The most common indication for surgical treatment was idiopathic osteoarthritis of the hip - 19 patients (Figures 1 and 2). The second group consisted of patients with degenerative changes based on sterile femoral head necrosis - 8 patients - AVN (Figures 3 and 4).

In surgical treatment, Hardinge's anterolateral access was used in positioning the patient on his/her back using a femoral neck endoprosthesis in full ceramic articulation.In patients clinical condition on the HHS, WOMAC-HIP scale, quality of life on the SF-12 scale and pain on the VAS scalewere assessed. Patients were evaluated before surgery.A control examination was performed after an average of 5 years.

The examination was conducted in accordance with the Declaration of Helsinki (1964) and its protocol was approved by the Local Bioethical Commission of the Medical University of Silesia in Katowice, Poland. All qualified patients signed a written consent for participation in this study.

\section{Statistical analysis}

Statistical analysis was performed using the Statistica 7.1 PL program. Measurable variables values are presented as the arithmetic mean with standard deviation. The normality of the distribution was checked by the Shapiro-Wilk test and the homogeneity of variance by the Levene test.Comparative analysis for groups was performed using the $t$ test for independent samples and the Mann-Whitney U test.Differences at the significance level $p<0.05$ were considered statistically significant. 


\section{Results}

In the study, clinical analysis on the hip joint of male patients using the following assessment scales: HHS and WOMAC-HIPwas performed.Analyzing the components of the HHS classification, i.e. pain and hip function, a statistically significant improvement in the control examination in terms of both assessed parameters $(p<0.05)$ was observed (Table No. 1$)$.

The stiffness, pain and activity of patients included in the WOMAC-HIP scale also showed statistical significance in the same observation period $(p<0.05)$ (Table 2$)$.

Comparing the results obtained in the HHS and WOMAC-HIP scales between the right and left side, no statistically significant differences $(p>0.05)$ were observed.

In addition, the study assessed the quality of life on the SF-12 scale and pain on the VAS scale.The total number of points on the SF-12 scale during the control examination was statistically significantly higher compared to the pre-surgery values $(p<0.05)$. The assessed pain on the VAS scale decreased statistically significantly in the control study compared to the value before surgery $(p<0.05)($ Table 3$)$.

\section{Discussion}

When choosing the right endoprosthesis for the patient, in addition to medical indications, age, gender, physical and professional activity of patients are also important.Considering the increasingly younger patients qualified for hip arthroplasty and their growing expectations in terms of activity and quality of life, femoral neck endoprosthesis may be increasingly used. This technique allows relatively high osteotomy in the femoral neck, with maximum bone tissue saving.However, this method also has its limitations and strict indications. Itcan not always be applied in situations of changed geometry of the femoral neck.Despite the lower risk of dislocation of the endoprosthesis, after this type of surgery there is a real risk of fracture of the femoral neck. Preliminary results of clinical trials are strongly encouraging, as they indicate a great opportunity to undertake physical and professional activity.It can also be used in very young patients with osteoarthritis of the hip due to sterile femoral head necrosis resulting from the use of steroid drugs in the treatment of autoimmune diseases [6-8].

Tsitlakidis S. et al. after analyzing available literature ( 27 works) on the assessment of the clinical status of patients with osteoarthritis of the hip, in whom the femoral neck endoprothesis was used in the proximal femoral end of the hip revealed, too short average survival of the implant (below 10 years) was foundAs the main reason for failure (revision), the authors pointed to aseptic loosening of the femoral stemand gave two main conditions for the success of this method: lopsided femoral neck and normal bone density [9].Our experience also shows that abnormal femoral neck geometry can affect a higher incidence of aseptic loosening in the early postoperative period. Tsitlakidis and our conclusions indicate that further observation of patients after femoral stemimplantation in hip arthroplasty is necessary and careful selection of patients qualified for this type of surgery is important. 
Used anterior - lateral access is considered as short cut access. There is a lot of data indicating that a minimally invasive procedure shortens hospitalization, enables faster rehabilitation and an earlier return to full physical activity.lt is also associated with less blood loss and less postoperative pain and a lower risk of infection [10-12].Confirmation of the above observations is the work of Xie J. et al. who showed that the type of surgical access in total hip arthroplasty closely correlates with pain experienced by the operated patients, measured by the VAS scale. Comparing the aforementioned parameter using the above-mentioned evaluation scale after a week, a month, 3 months and a year in equal groups of patientsoperated on using the posterior access method and minimally invasive supracapsulartransdermally assisted method, the authors showed a significant reduction in the second of the examined groups. Therefore, one can cite the authors of the study that minimally invasive supracapsular access transdermally assisted in hip arthroplasty results in a significant reduction of pain sensations from the time of surgery up to 3 months compared to pain experienced after classic posterior access in the same assessment periods. A lower level of pain sensations within a period of up to 3 months after implantation of the hip joint prosthesis should directly affect the earlier physiotherapy after surgery and shorten the period of stay in the ward, while allowing the possibility of earlier independent existence after surgery [13].In turn, in our study using anterolateral access according to Hardinge'a, we obtained a reduction in pain on the VAS scale of 0.58 points, in the assessment carried out after an average of 5 years. Therefore, the result obtained by us seems to be fully satisfactory.

Constantly rising expectations of patients with advanced cokearthrosis, their young age and willingness to return to full activity forces engineers-constructors and orthopedic physicians to seek ever newer solutions in hip replacement arthroplasty aimed at developing the concept of ultra-short cementlessstem. The authors, performing densitometry using the DXA method and assessing Radiostereometric Analysis (RSA) of the PRIMOR implant in 50 patients operated on within 2 years of surgery, found its settling 6 weeks after surgery and clubbing occurring between 6 and 12 months after arthroplasty.They found the test results to be satisfactory, showing a positive correlation between better bone quality and lower stemimplant migration in the proximal femur in operated patients [14].

The mere use of new technologies and structures is not enough to ensure a lasting, beneficial effect of the treatment.The success of arthroplasty still depends primarily on the experience of the surgical team performing the surgery; while access to modern techniques and implants allows to improve patients' quality of life $[3,15]$.

Birkhauer B. et al. in their work in a group of 38 patients over 60 years of age using the SPIRON stem neck improved their clinical condition in the early observation period (over 1 year) on the HHS scale (24 points vs. 78 points), average - 94 points. The authors performed only one revision surgery within 3 months of surgery due to early joint infection (2.63\%) [16].

In turn, Lugeder A. et al. on a group of 28 patients observed a significant improvement on the HHS scale (55.4 vs 90.5) after 3 months after surgery. Only in one case, there was aseptic loosening of the stem component and a revision surgery had to be performed (3.6\%). In both of the above works, the authors 
made a clinical evaluation in the early observation period ( 3 months, 1 year) and obtained very good results [17]. Our assessment was carried out after an average of 5 years after surgery.We obtained a slightly lower result (average 85.4 points) in the clinical assessment of HHS. In the examined group of patients, we also noted a higher percentage of revision procedures performed in three patients $(11 \%)$ due to a defective implanted SPIRON stem (deformed).In two patients during revision surgery,the CorinMiniHipparanasalstemwas used, and in one case the classic cementlessCorrail J \& J DePuy.

Among other complications that occurred after surgery, we found deep vein thrombosis ( 1 patient - 3.7\%) and transient femoral nerve paresis (1 patient - 3.7\%).The higher number of revisions probably resulted from the etiology of degenerative disease and worse conditions for proper - axial implantation of the endoprosthesis stem.In almost 1/3 (8 operated patients), the cause of the degenerative disease was sterile femoral head necrosis, which significantly worsened the conditions for femoral stem deposition.

Pyda M. et al. using surface arthroplasty in 30 patients with sterile femoral head necrosis achieved clinical improvement of over 94 points (on the HHS scale), improving the level of physical activity to 7.55 points (UCLA) in the mean follow-up of 7 years after surgery. The authorsdid not noteloosening of the endoprosthesiscomponents [18].

WhereasAmstutz H.C. et al. in the medium follow-up period (10.8 years) confirmed a significantimprovement in the clinicalcondition in a group of 82 patientsafter the application of surfacearthroplasty in the course of hip osteoarthritisbased on sterilefemoralosteonecrosis [15].

Keeney J.A. et al. performed retrospective pre- and postoperative demographic characteristics and functional activity profiles using classic assessment scales in two groups; under $<50$ years and $>65<75$ years in the mean follow-up period of a minimum of 1 year (12 months). The authors, analyzing the postoperative results of clinical condition assessment between the examined groups, did not find any significant differences. However, in the assessment of post-operative functional activity measured by UCLA classification, $37 \%$ of the operated in the group up to 50 years of age and $15.5 \%$ in the group between 65 and 75 years old returned to the previously implemented activity. The above authors concluded that the younger ones operated on after hip replacement are likely to return to high levels of functional activity.In turn, high levels of functional activity are less popular in younger patients with diagnoses other than osteoarthritis.In addition, the age of the operatedpatientsis not a simplesubstitute for the level of functionalactivity in patientsconsidering hip arthroplasty [19].

Cowie J.G. et al. in 239 patients, assessing the impact of hip replacement in relation to professional and sport activity, showed the possibility of their resumption after a minimum of 4-6 months after the surgery.In addition, they found that higher BMI in operated patients extended this time period [20].

In turn, Czech Sz. et al. in a group of 106 operated patients who were treated with the short metaphyseal stem. Nanos found a very beneficial effect of its application in the field of physical activity. At the same time, the authors drew attention to the decreasing level of physical activity in patients operated on with age [21]. 
Oken F.O. et al. analyzed 51 professionally active patients under the age of 60 who had hip arthroplasty during developmental dysplasia.The authors found a beneficial effect of arthroplasty on early return to work for most patients. The status of the unemployed who are ready to take up employment has also changed.In addition, carrying out endoprostheoplastyin the above-mentionedpatientsincreased the economic status of the region in whichtheyworked [22].

The constantly growing number of employed patients qualified for hip replacement requires knowledge of the factors conditioning their return to work after performing the surgery and undergoing the necessary rehabilitation. The authors analyzed a group of 408 patients employed in the public sector at an average age of 54 ; of which $73 \%$ of the total operated patients were women. $94 \%$ of patients employed before surgery returned to work after an average of 3 months after surgery. The identified significant risk factors for returning to work were: absence shorter than 30 days prior to surgery, occupied senior position and $\mathrm{BMI}$ less than 30 . Factors such as age, gender, pre-operative health, and various health-related behaviors did not show any dependence on returning to work after endoprosthesis implantation. Obese manual workers with a period of absence from work before the endoprosthesis of more than 30 days constituted a group of patients at higher risk of not returning to work after surgery [4].

Fisher N.E. et al. evaluated sport and physical activity in 117 patients after hip arthroplasty in a short observation period ( 2 years), showing that $87 \%$ of the patients returned to physical and sport activity before surgery [23].

Perneger T.V. et al. assessed the quality of life with the SF-12 classification in patients undergoing hip and knee replacement. The tests were carried out before surgery and one year after it. In the analysis, the authors pointed to a significant increase in parameters on the SF-12 scale.However, it should be noted that the numerical value of the PCS components during the control test after one year was significantly higher than the MCS components.According to the authors, carrying out hip or knee arthroplasty has a positive effect on improving the quality of life in patients operated on both in the mental and physical sphere, although the more favorable impact of performing this procedure is more visible on a physical level [24].

The analysis carried out above shows that femoral neck endoprosthesis should be an alternative to pituitary and classical cementlessarthroplasty.The correct qualification of patients for this type of surgery is crucial and very important. However, it requires more attention from the surgeon when implanting this type of stem in the femoral neck with altered geometry.

\section{Conclusions}

1. The performance of femoral neck endoprosthesis improved the clinical condition and quality of life of the operated patients.

2. The use of the SPIRON femoral neck endoprosthesis reduced pain. 


\section{List Of Abbreviations}

HHS - Harris Hip Score

WOMAC-HIP - Western Ontario and McMasterUniversitiesArthritis Index of HiP

SF-12 - Short Form SF - 12

VAS - Visual AnalogueScale

AVN - AvascularNecrosis

FAl - Femoroacetabularimpingement

UCLA - UCAL LonelinessScale

BMI - Body Mass Index

PCS - Physical Component Score

MCS - Mental Component Score

\section{Declarations}

\section{Ethics approval and consent to participate}

The examination was conducted in accordance with the Declaration of Helsinki (1964) and its protocol was approved by the Local Bioethical Commission of the Medical University of Silesia in Katowice, Poland (permission no.: KNW/110/11). All qualified patients signed a written consent for participation in this study.

\section{Consent for publication}

Not applicable.

\section{Availability of data and materials}

The datasetsused and/oranalysedduring the currentstudyareavailable from the correspondingauthor on reasonablerequest.

\section{Competing interests}

The author's declare that there is no conflict of interests regarding the publication of this paper.

\section{Funding}


Not applicable.

\section{Authors' contributions}

TS - study design, data collection, data interpretation, manuscript preparation

JP - study design, data collection, data interpretation, manuscript preparation

MP - study design, data collection, data interpretation, manuscript preparation

DS - data collection, data interpretation, manuscript preparation, statistical analysis

MB - data collection, manuscript preparation, literature search

$\mathrm{BD}$ - data collection, literature search

WG - data interpretation, manuscript preparation

$\mathrm{MCz}$ - data interpretation, manuscript preparation

BK - data collection, literature search

\section{Acknowledgements}

Not applicable.

\section{References:}

1. Bergman J, Nordström A, Nordström P. Epidemiology of osteonecrosis among older adults in Sweden. OsteoporosInt. 2019;30(5):965-73.

2. 2. Shakoor $A B$, Baig MN. Osteonecrosis of the Femoral Head: Etiology, Investigations, and Management Cureus. 2018;10(8):31-71.

3. Asaad A, Hart A, Michael M.Y. Khoo B, Kevin I et al. Frequent Femoral Neck Osteolysis With Birmingham Mid-head Resection Resurfacing Arthroplasty in Young Patients. ClinOrthopRelat Res. 2015;473:3770-78.

4. Laasik R, Lankinen P, Kivimäki M, Aalto V, Saltychev M et al. Return to work after primary total hip arthroplasty: a nationwide cohort study. Acta Orthop. 2019;90(3):209-13.

5. Takashima K, Sakai T, Hamada H, Takao M, Sugano N. Which Classification System Is Most Useful for Classifying Osteonecrosis of the Femoral Head? ClinOrthopRelat Res. 2018;476(6):1240-49.

6. Houdek MT, Wyles CC, Collins MS, Howe BM, Terzic A et al. Stem Cells Combined With Platelet-rich Plasma Effectively Treat Corticosteroid-induced Osteonecrosis of the Hip: A Prospective Study. ClinOrthopRelat Res. 2018;476(2):388-97. 
7. Lerch TD, Vuilleumier S, Schmaranzer F, Ziebarth K, Steppacher SD et al. Patients with severe slipped capital femoral epiphysis treated by the modified Dunn procedure have low rates of avascular necrosis, good outcomes, and little osteoarthritis at long-term follow-up. Bone Joint J. 2019;101$B(4): 403-14$.

8. Luo RB, Lin T, Zhong HM, Yan SG, Wang JA. Evidence for using alendronate to treat adult avascular necrosis of the femoral head: a systematic review. MedSci Monit. 2014;20:2439-47.

9. Tsitlakidis S, Westhauser F, Horsch A, Beckmann N, Bitsch R, and Matthias Klotz. Femoral neck prostheses: A systematic analysis of the literature. Orthop Rev (Pavia). 2019;11(3):8204.

10. Derek J, McMinn W, PradhanCh, Ziaee H, Daniel J. Is Mid-head Resection a Durable Conservative Option in the Presence of Poor Femoral Bone Quality and Distorted Anatomy? ClinOrthopRelat Res. 2011;469:1589-97.

11. Adeel A, Sheikh HQ, Masjedi M, Jeffers J, Cobb J. Birmingham Mid-Head Resection Periprosthetic Fracture. Clinics in OrthopedicSurgery. 2015;7:402-05.

12. Rahman L, Sarah N, Mvirhead A. The Birmingham Mid-Head Resection Arthroplasty - Minimum Two Year Clinical and Radiological Follow-Up: An Independent Single Surgeon Series. BMHR. 2015;17(4):225-29.

13. Xie J, Zhang H, Wang L, Yao X, Zhanpeng P, Qinyi J. Comparison of supercapsularpercutaneously assisted aprroach total hip versus conventional posterior approach for total hip arthroplasty: a. prospective, randomisedconrolled trial. Journal of OrthopaedicSurgery and Research. 2017;12:13843.

14. Christiansen JD, Ejaz A, Nielsen PT, Laursen M.An Ultra-Short Femoral Neck-Preserving Hip Prosthesis: A 2-Year Follow-up Study with RadiostereometricAnalysis and Dual X-Ray Absorptiometry in a Stepwise Introduction.J Bone Joint Surg Am. 2020;102(2):128-36.

15. Amstutz HC, Le Duff MJ. Hip resurfacing for osteonecrosis: two- to 18-year results of the Conserve Plus design and technique. J Bone Joint Surg Br. 2016;98:901-09.

16. Birkenhauer B, Kistmacher H, Ries

J.ZementfreieSchenkelhalsschraubenprotheseTypSpironKonzeptionundersteklinischeErgebnisse. Orthopäde. 2004;33:1259-66.

17. Pyda M, Koczy B, Widuchowski W, Widuchowska M, Stołtny T et al.Hip Resurfacing Arthroplasty in Treatment of Avascular Necrosis of the Femoral Head. MedSci Monit. 2015;21:304-09.

18. Lugeder A, Häring E, Müller A, Droste P, Zeichen J. Hüftgelenk ersatz mit der zementfreienSpironSchenkelhalsprothese. Oper OrthopTraumatol. 2013;25: 388-97.

19. Keeney JA, Nunley RM, Baca GR, Clohisy JC. Are younger patients undergoing THA appropriately characterized as active? ClinOrthopRelat Res. 2015;473(3):1083-92.

20. Cowie JG, Turnbull GS, Ker AM, Breusch SJ. Return to work and sports after total hip replacement. Arch Orthop Trauma Surg.2013;133(5):695-700.

21. Czech Sz, Hermanson J, Kasperczyk S, Białkowska M, Kokot R et al.Cementless neck preserving arthroplasty should be the treatment of choice in cases of coxarthrosis in middle-aged men: very 
good results in a mid-term follow-up study with NANOS stem and Verilast articulation. ChirNarzadow Ruchu Ortop Pol. 2018;83(3):103-07.

22. Oken FO, Yildirim OA, Asilturk M. Factors affecting the return to work of total hip arthroplasty due to of developmental hip dysplasia in in young patients. J Orthop. 2018;15(2):450-54.

23. Fisher NE, Killampalli VV, Kundra RK, Jagodzinski NA, Mathur K, Reading AD. Sporting and physicalactivityfollowing hip resurfacing. 2011;35(7):977-80.

24. Perneger TV, Hannouche D, Miozzari HH, Lubbecke AA.Sympthoms of osteoarhritis influence mental and physical health differently before and after joint replacement surgery: A prospective study. PloS ONE. 2019;14(6):21-27.

25.

\section{Tables}

Table 1.

Results of clinical evaluation of men on the HHS scale before and after SPIRON femoral neck endoprosthesis.

\begin{tabular}{|llll|}
\hline HHS rightside & Beforesurgery & Aftersurgery & $\mathrm{p}$ \\
\hline pain & 13,68 & 39,9 & $<0.05$ \\
\hline function & 25,05 & 41,75 & $<0.05$ \\
HHS leftside & Beforesurgery & Aftersurgery & $\mathrm{P}$ \\
\hline pain & 12,26 & 42,88 & $<0.05$ \\
\hline function & 24,81 & 44,83 & $<0.05$ \\
\hline
\end{tabular}


Table 2.

Results of the clinical evaluation of men on the WOMAC-HIP scale before and after SPIRON femoral neck endoprosthesis.

\begin{tabular}{|llll|}
\hline WOMAC rightside & Beforesurgery & Aftersurgery & $\mathrm{p}$ \\
\hline stiffness & 3,68 & 0,78 & $<0.05$ \\
\hline pain & 12,73 & 1,05 & $<0.05$ \\
\hline activity & 37,36 & 7,09 & $<0.05$ \\
\hline WOMAC leftside & Beforesurgery & Aftersurgery & $\mathrm{P}$ \\
\hline stiffness & 3,37 & 0,31 & $<0.05$ \\
\hline pain & 11,5 & 1,75 & $<0.05$ \\
\hline activity & 29,93 & 8,68 & $<0.05$ \\
\hline
\end{tabular}

Table 3.

Results of quality of life assessment on the SF-12 scale and pain on the VAS scale of men after SPIRON femoral neck endoprosthesis.

\begin{tabular}{|llll|}
\hline & Beforesurgery & Aftersurgery & $\mathrm{p}$ \\
\hline SF-12 & 9,89 & 32 & $<0.05$ \\
\hline VAS & 6,74 & 0,58 & $<0.05$ \\
\hline
\end{tabular}

\section{Figures}




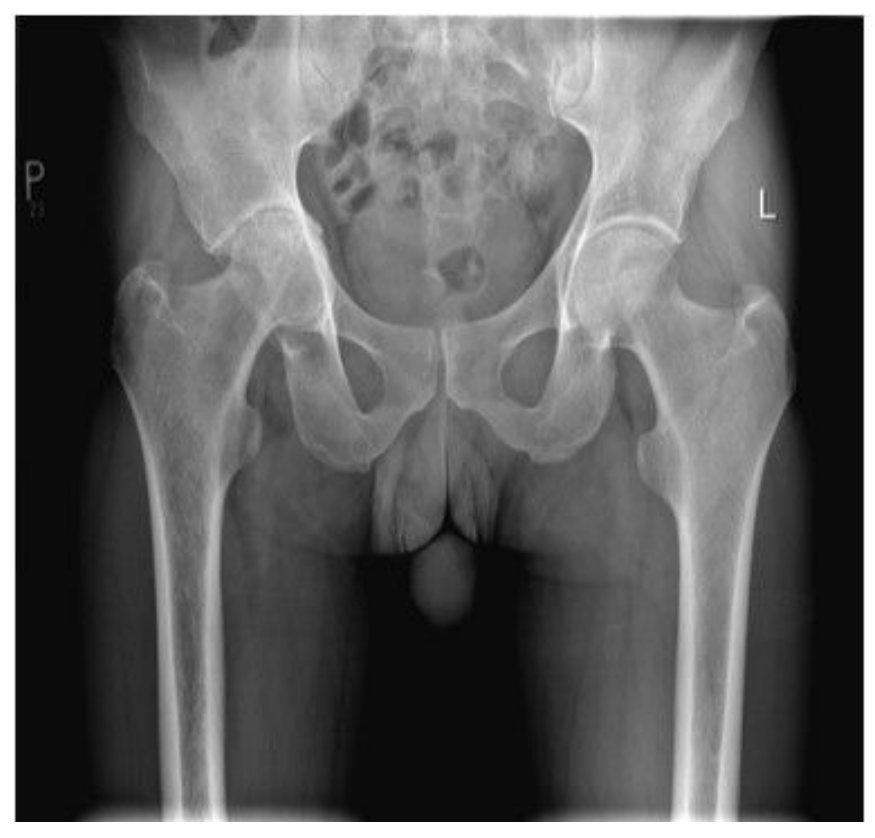

\section{Figure 1}

Patient 42 years old diagnosed with right-sided coxartrosis with head protrusion into the pelvis.

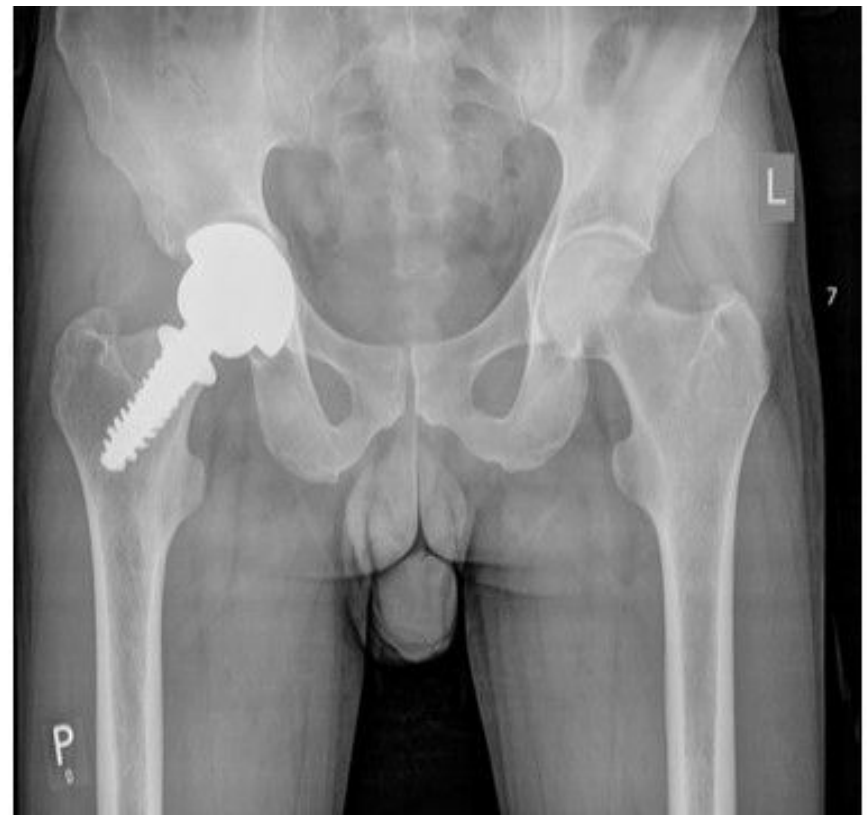

\section{Figure 2}

Patient 42 years old after SPIRON femoral neck endoprosthesis. 


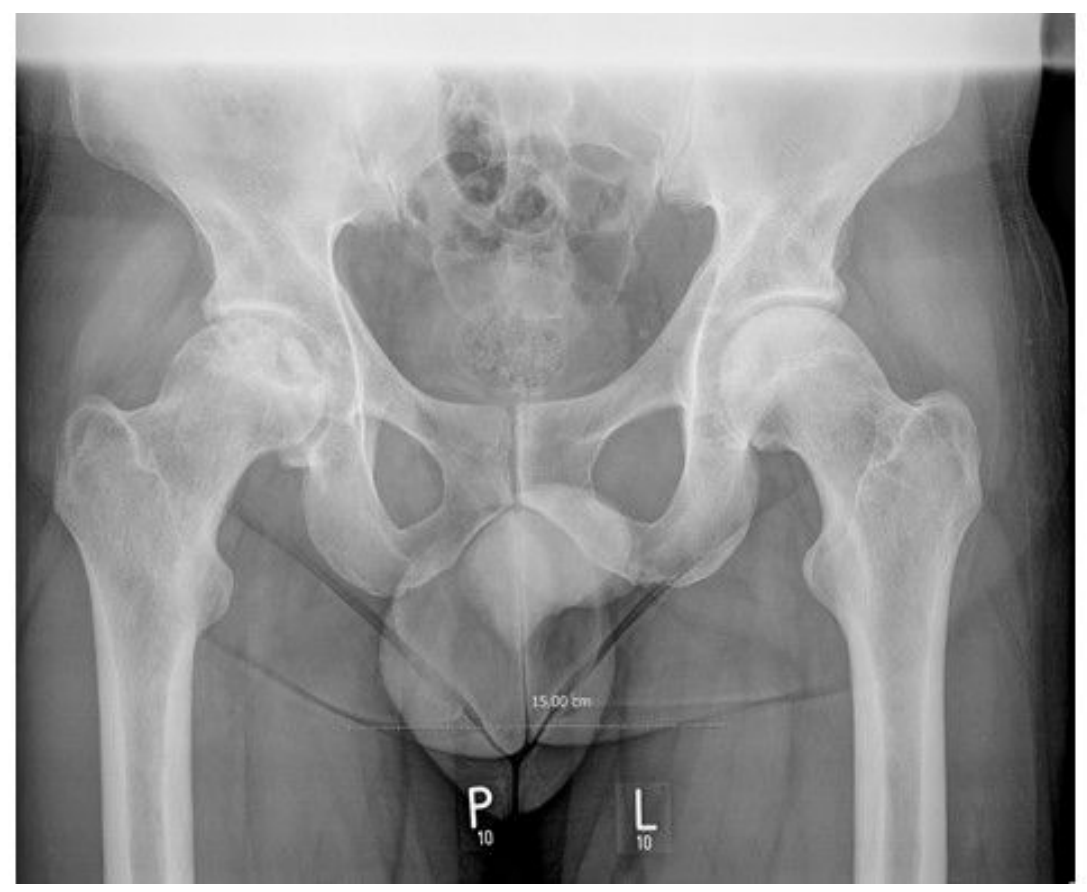

\section{Figure 3}

Patient 54 years old patient diagnosed with bilateral degenerative changes of the hip joints along the length of avascular necrosis (AVN) on the right and on the left side of FAl.

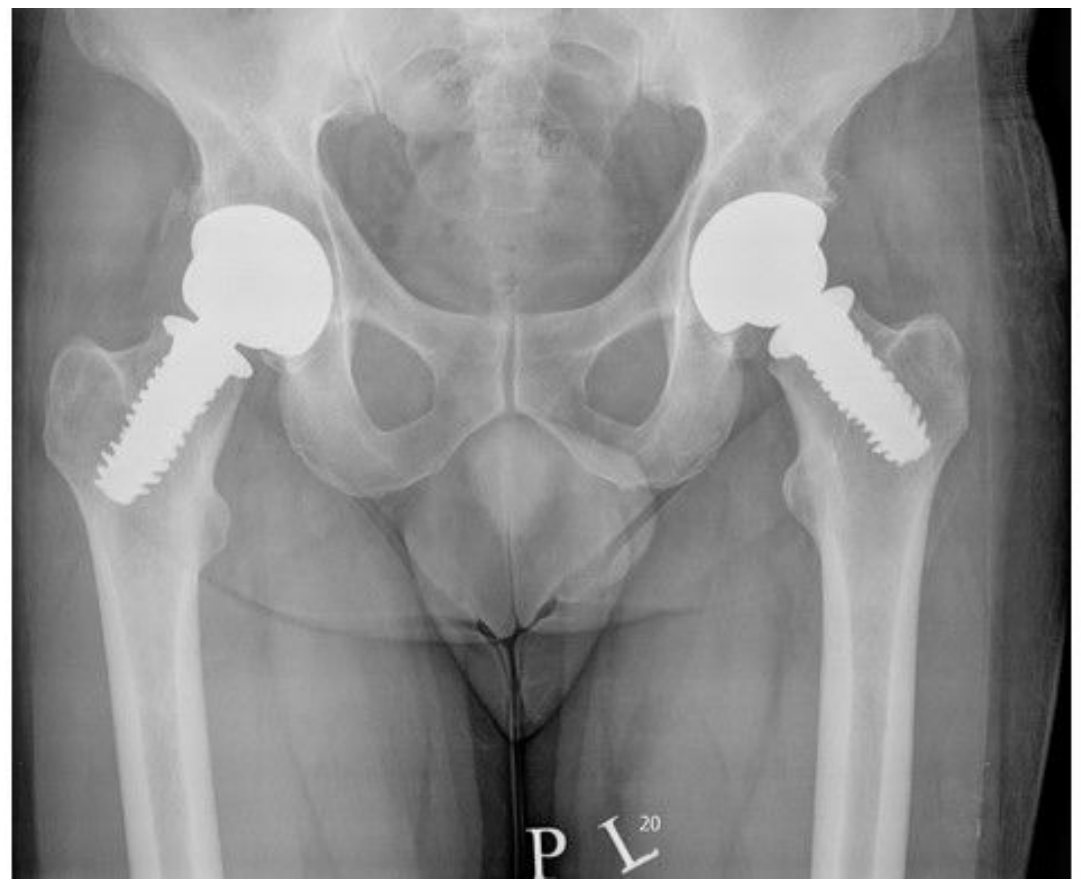

\section{Figure 4}

Patient 54 years old after bilateral hip arthroplasty with the SPIRON method. 


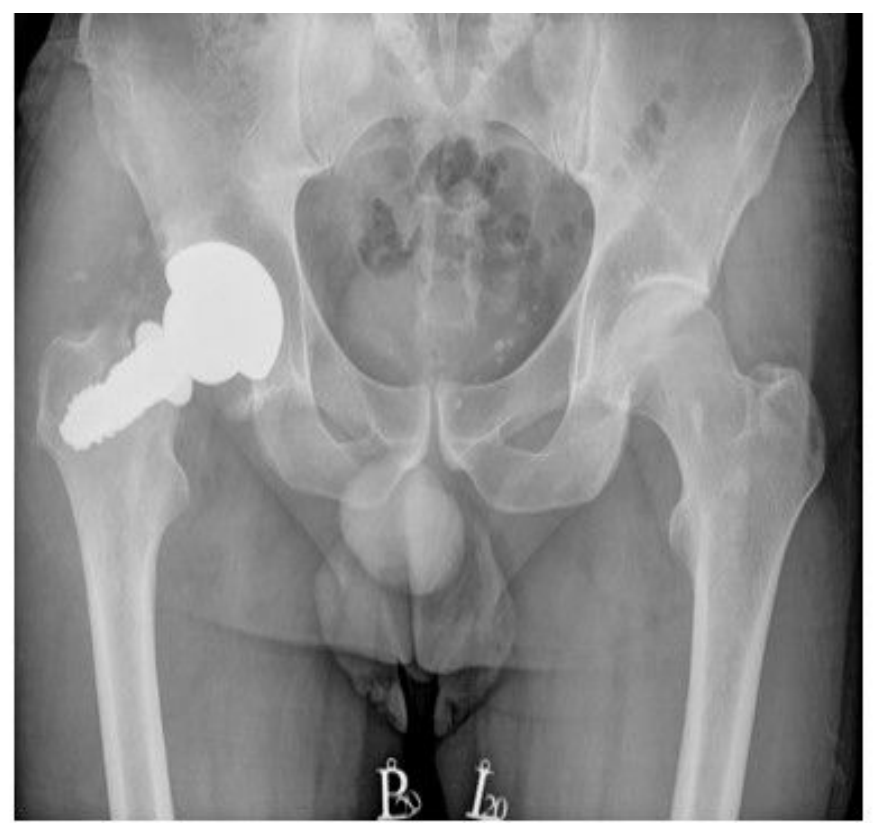

Figure 5

Patient 54 years old with defective (deformed) SPIRON stem prior to hip revision surgery.

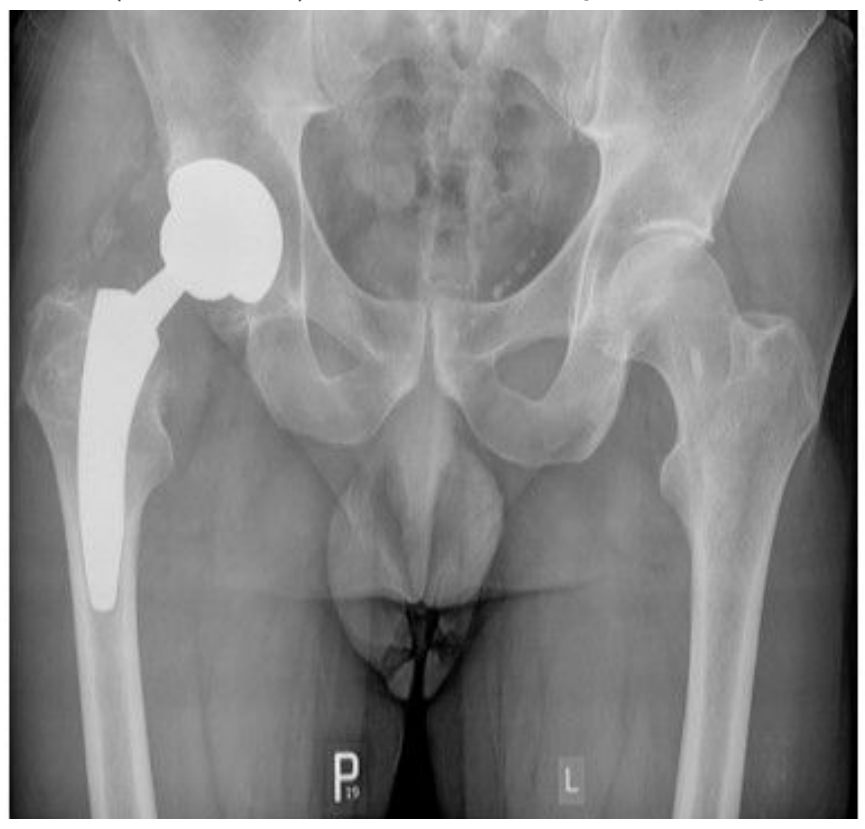

Figure 6

Patient 54 years old after hip revision surgery with the CorinMiniHip puncture stem. 